\title{
Fractional differential equations with a Caputo derivative with respect to a kernel function and their applications
}

\author{
Ricardo Almeida ${ }^{1 *}$, Agnieszka B. Malinowska ${ }^{2}$, and M. Teresa T. \\ Monteiro $^{3}$
}

This paper is devoted to the study of the initial value problem of nonlinear fractional differential equations involving a Caputo type fractional derivative with respect to another function. Existence and uniqueness results for the problem are established by means of the some standard fixed point theorems. Next, we develop the Picard iteration method for solving numerically the problem, and obtain results on the long-term behaviour of solutions. Finally, we analyze a population growth model and a gross domestic product model with governing equations being fractional differential equations that we have introduced in this work. Copyright (c) 2009 John Wiley \& Sons, Ltd.

Keywords: Fractional calculus, fractional differential equations, population growth model, gross domestic product model

\section{Introduction}

Fractional calculus is a branch of mathematics that studies integrals and derivatives of non-integer order. Leibniz and L-Hôpital have already wondered what would be a derivative of order 0.5 . Although in the beginning fractional calculus had a development as a pure mathematical idea, in recent decades its use has also spread into many other fields of science such as physics, mechanics, and bioengineering $[13,19]$. Various phenomena of viscoelasticity, diffusion procedures, relaxation vibrations, electrochemistry, etc. are successfully described by fractional differential equations (FDE). The researchers tried to suggest several types of fractional operators to describe more accurately these phenomena (see, e.g., $[1,6,7,22,28]$ ). Due to the large number of definitions that exist for fractional derivatives, one simple way to deal with such a variety is to combine those concepts to a single one by considering fractional derivatives of function $f$ with respect to another function [21]. Moreover, as the purpose operator depends on a kernel, it provides, besides the order, any number of free parameters to better calibrate a system. Therefore, we find the theory of FDEs with derivatives depending on a kernel, as a promising area for further investigations. In this paper, we intend to present the fundamentals of a theory for FDEs with a general derivative. For related results concerning FDEs with different type of fractional derivatives, we refer to $[2,3,4,9,10,17,25]$.

The outline of the paper is the following. In Section 2, we present the main definition of this work: the $\psi$-Caputo fractional derivative, that is, a Caputo-type derivative of a function with respect to another function; in Theorem 1, we prove that this operator is the left inverse of the fractional integral. Section 3 is devoted to the study of FDEs with $\psi$-Caputo fractional derivatives. First, in Section 3.1, the problem of existence and uniqueness of solutions is investigated. Then, based on the Picard iteration method, we present a scheme for solving this type of FDEs. Section 3.3 contains results on the long-term behaviour of solutions defined on sufficiently large intervals. In Section 4, possible applications of the theory developed in this paper to

${ }^{1}$ Center for Research and Development in Mathematics and Applications (CIDMA)

Department of Mathematics, University of Aveiro, 3810-193 Aveiro, Portugal

${ }^{2}$ Faculty of Computer Science

Bialystok University of Technology, 15-351 Białystok, Poland

${ }^{3}$ Algoritmi R\&D Center, Department of Production and Systems

University of Minho, Campus de Gualtar, 4710-057 Braga, Portugal

* Correspondence to: Ricardo Almeida, University of Aveiro, Aveiro, Portugal. E-mail: ricardo.almeida@ua.pt 
model a population growth and a gross domestic product are presented.As governing equations of a mathematical formulation of those models we propose fractional differential equations with the $\psi$-Caputo fractional derivative. Finally, we finish the paper by a conclusion section

\section{Preliminaries}

In the following and throughout the text, $\alpha>0$ is a real, $x:[a, b] \rightarrow \mathbb{R}$ an integrable function and $\psi \in C^{n}[a, b]$ an increasing function such that $\psi^{\prime}(x) \neq 0$, for all $x \in[a, b]$.

The $\psi$-Riemann-Liouville fractional integral of $x$ of order $\alpha$ is defined as

$$
l_{a+}^{\alpha, \psi} x(t):=\frac{1}{\Gamma(\alpha)} \int_{a}^{t} \psi^{\prime}(\tau)(\psi(t)-\psi(\tau))^{\alpha-1} x(\tau) d \tau,
$$

and the $\psi$-Riemann-Liouville fractional derivative of $x$ of order $\alpha$ is defined as

$$
\begin{aligned}
D_{a+}^{\alpha, \psi} x(t) & :=\left(\frac{1}{\psi^{\prime}(t)} \frac{d}{d t}\right)^{n} I_{a+}^{n-\alpha, \psi} x(t) \\
& =\frac{1}{\Gamma(n-\alpha)}\left(\frac{1}{\psi^{\prime}(t)} \frac{d}{d t}\right)^{n} \int_{a}^{t} \psi^{\prime}(\tau)(\psi(t)-\psi(\tau))^{n-\alpha-1} x(\tau) d \tau .
\end{aligned}
$$

Here, $n=[\alpha]+1$. In particular, for suitably chosen $\psi$, we obtain some well known fractional operators, like the RiemannLiouville, the Hadamard and the Erdélyi-Kober type. The fractional integrals satisfy the semigroup law [15]: let $\alpha, \beta>0$, then the relation

$$
\left.l_{a+}^{\alpha, \psi}\right|_{a+} ^{\beta, \psi} x(t)=l_{a+}^{\alpha+\beta, \psi} x(t)
$$

holds. In the present work, we deal with a Caputo type differential operator.

Definition 1 Let $\alpha>0$ and $\psi \in C^{n}[a, b]$ be a function such that $\psi$ is increasing and $\psi^{\prime}(x) \neq 0$, for all $x \in[a, b]$. Given $x \in C^{n-1}[a, b]$, the $\psi$-Caputo fractional derivative of $x$ of order $\alpha$ is defined as

$$
{ }^{c} D_{a+}^{\alpha, \psi} x(t):=D_{a+}^{\alpha, \psi}\left[x(t)-\sum_{k=0}^{n-1} \frac{x_{\psi}^{[k]}(a)}{k !}(\psi(t)-\psi(a))^{k}\right]
$$

where

$$
n=[\alpha]+1 \text { for } \alpha \notin \mathbb{N}, \quad n=\alpha \text { for } \alpha \in \mathbb{N},
$$

and

$$
x_{\psi}^{[k]}(t):=\left(\frac{1}{\psi^{\prime}(t)} \frac{d}{d t}\right)^{k} x(t)
$$

If $x \in C^{n}[a, b]$, then the $\psi$-Caputo fractional derivative of $x$ can be represented by the expression (cf. [5, Theorem 3])

$$
{ }^{c} D_{a+}^{\alpha, \psi} x(t):=l_{a+}^{n-\alpha, \psi}\left(\frac{1}{\psi^{\prime}(t)} \frac{d}{d t}\right)^{n} x(t) .
$$

Thus, if $\alpha=m \in \mathbb{N}$, we have

$$
{ }^{C} D_{a+}^{\alpha, \psi} x(t)=x_{\psi}^{[m]}(t)
$$

and for $\alpha \notin \mathbb{N}$, we have

$$
{ }^{c} D_{a+}^{\alpha, \psi} x(t)=\frac{1}{\Gamma(n-\alpha)} \int_{a}^{t} \psi^{\prime}(\tau)(\psi(t)-\psi(\tau))^{n-\alpha-1} x_{\psi}^{[n]}(\tau) d \tau .
$$

Some known fractional derivatives are just particular cases of the $\psi$-Caputo fractional derivative. For appropriate choices of the kernel $\psi$, we obtain the Caputo fractional derivative [21] (for $\psi(t)=t$ ), the Caputo-Hadamard fractional derivative [11, 14] (for $\psi(t)=\ln (t)$ ) and the Caputo-Erdélyi-Kober fractional derivative [18] (for $\psi(t)=t^{\sigma}$ ).

The $\psi$-Caputo fractional derivative of a power function is given next. Let $\beta \in \mathbb{R}$ with $\beta>n$. The $\psi$-Caputo fractional derivative of the function

is given by the formula

$$
x(t)=(\psi(t)-\psi(a))^{\beta-1}
$$

$$
{ }^{c} D_{a+}^{\alpha, \psi} x(t)=\frac{\Gamma(\beta)}{\Gamma(\beta-\alpha)}(\psi(t)-\psi(a))^{\beta-\alpha-1} .
$$

The $\psi$-Caputo fractional derivative is a left inverse of the $\psi$-Riemann-Liouville fractional integral. Below we generalize Theorems 4 and 5 of [5]. 
Theorem 1 Let $x:[a, b] \rightarrow \mathbb{R}$. The following holds:

1. If $x \in C[a, b]$, then

$$
{ }^{C} D_{a+}^{\alpha, \psi} I_{a+}^{\alpha, \psi} x(t)=x(t) .
$$

2. If $x \in C^{n-1}[a, b]$, then

$$
I_{a+}^{\alpha, \psi C} D_{a+}^{\alpha, \psi} x(t)=x(t)-\sum_{k=0}^{n-1} \frac{x_{\psi}^{[k]}(a)}{k !}(\psi(t)-\psi(a))^{k} .
$$

Proof For proving 1 observe that, by definition,

$$
{ }^{c} D_{a+}^{\alpha, \psi} I_{a+}^{\alpha, \psi} x(t):=D_{a+}^{\alpha, \psi}\left[l_{a+}^{\alpha, \psi} x(t)-\sum_{k=0}^{n-1} \frac{\left(I_{a+}^{\alpha, \psi} x\right)_{\psi}^{[k]}(a)}{k !}(\psi(t)-\psi(a))^{k}\right] .
$$

Attending that

$$
\begin{aligned}
\left(I_{a+}^{\alpha, \psi} x\right)_{\psi}^{[k]}(t) & =\left(\frac{1}{\psi^{\prime}(t)} \frac{d}{d t}\right)^{k} l_{a+}^{\alpha, \psi} x(t) \\
& =\frac{1}{\Gamma(\alpha-k)} \int_{a}^{t} \psi^{\prime}(\tau)(\psi(t)-\psi(\tau))^{\alpha-k-1} x(\tau) d \tau
\end{aligned}
$$

we deduce the following relation

$$
\left|\left(I_{a+}^{\alpha, \psi} x\right)_{\psi}^{[k]}(t)\right| \leq \frac{\|x\|}{\Gamma(\alpha-k+1)}(\psi(t)-\psi(a))^{\alpha-k},
$$

and thus $\left(I_{a+}^{\alpha, \psi} x\right)_{\psi}^{[k]}(a)=0$, for all $k=0,1, \ldots, n-1$. Therefore,

$$
{ }^{c} D_{a+}^{\alpha, \psi} I_{a+}^{\alpha, \psi} x(t)=D_{a+}^{\alpha, \psi} I_{a+}^{\alpha, \psi} x(t)=\left(\frac{1}{\psi^{\prime}(t)} \frac{d}{d t}\right)^{n} I_{a+}^{n-\alpha, \psi} I_{a+}^{\alpha, \psi} x(t)=\left(\frac{1}{\psi^{\prime}(t)} \frac{d}{d t}\right)^{n} I_{a+}^{n, \psi} x(t)=x(t),
$$

which ends the first point of the proof. To prove 2, let

$$
y(t):=x(t)-\sum_{k=0}^{n-1} \frac{x_{\psi}^{[k]}(a)}{k !}(\psi(t)-\psi(a))^{k} .
$$

Thus, $\left.\right|_{a+} ^{\alpha, \psi C} D_{a+}^{\alpha, \psi} x(t)=l_{a+}^{\alpha, \psi} D_{a+}^{\alpha, \psi} y(t)$, and so it is enough to prove that $l_{a+}^{\alpha, \psi} D_{a+}^{\alpha, \psi} y(t)=y(t)$. For this purpose, observe that

$$
\begin{aligned}
l_{a+}^{\alpha, \psi} D_{a+}^{\alpha, \psi} y(t) & =\frac{1}{\Gamma(\alpha)} \int_{a}^{t} \psi^{\prime}(\tau)(\psi(t)-\psi(\tau))^{\alpha-1} D_{a+}^{\alpha, \psi} y(\tau) d \tau \\
& =\frac{1}{\psi^{\prime}(t)} \frac{d}{d t}\left\{\frac{1}{\Gamma(\alpha+1)} \int_{a}^{t} \psi^{\prime}(\tau)(\psi(t)-\psi(\tau))^{\alpha} D_{a+}^{\alpha, \psi} y(\tau) d \tau\right\} .
\end{aligned}
$$

Integrating by parts, we obtain

$$
\begin{aligned}
\star & :=\frac{1}{\Gamma(\alpha+1)} \int_{a}^{t} \psi^{\prime}(\tau)(\psi(t)-\psi(\tau))^{\alpha} D_{a+}^{\alpha, \psi} y(\tau) d \tau \\
= & \frac{1}{\Gamma(\alpha+1)} \int_{a}^{t}(\psi(t)-\psi(\tau))^{\alpha} \frac{d}{d \tau}\left[\left(\frac{1}{\psi^{\prime}(\tau)} \frac{d}{d \tau}\right)^{n-1} l_{a+}^{n-\alpha, \psi} y(\tau)\right] d \tau \\
= & {\left[\frac{(\psi(t)-\psi(\tau))^{\alpha}}{\Gamma(\alpha+1)}\left(\frac{1}{\psi^{\prime}(\tau)} \frac{d}{d \tau}\right)^{n-1} l_{a+}^{n-\alpha, \psi} y(\tau)\right]_{a}^{t} } \\
& +\frac{1}{\Gamma(\alpha)} \int_{a}^{t}(\psi(t)-\psi(\tau))^{\alpha-1} \frac{d}{d \tau}\left[\left(\frac{1}{\psi^{\prime}(\tau)} \frac{d}{d \tau}\right)^{n-2} l_{a+}^{n-\alpha, \psi} y(\tau)\right] d \tau .
\end{aligned}
$$

Since

$$
\left(\frac{1}{\psi^{\prime}(\tau)} \frac{d}{d \tau}\right)^{n-1} l_{a+}^{n-\alpha, \psi} y(\tau)=\frac{1}{\Gamma(1-\alpha)} \int_{a}^{\tau} \psi^{\prime}(s)(\psi(\tau)-\psi(s))^{-\alpha} y(s) d s,
$$

we deduce that

$$
\left|\left(\frac{1}{\psi^{\prime}(\tau)} \frac{d}{d \tau}\right)^{n-1} l_{a+}^{n-\alpha, \psi} y(\tau)\right| \leq \frac{\|y\|}{\Gamma(2-\alpha)}(\psi(\tau)-\psi(a))^{1-\alpha}
$$


and so

$$
\left(\frac{1}{\psi^{\prime}(\tau)} \frac{d}{d \tau}\right)^{n-1} l_{a+}^{n-\alpha, \psi} y(\tau)=0 \quad \text { at } \quad \tau=a .
$$

Thus, performing again integration by parts, we obtain the equality

$$
\begin{aligned}
\star= & \frac{1}{\Gamma(\alpha)} \int_{a}^{t}(\psi(t)-\psi(\tau))^{\alpha-1} \frac{d}{d \tau}\left[\left(\frac{1}{\psi^{\prime}(\tau)} \frac{d}{d \tau}\right)^{n-2} I_{a+}^{n-\alpha, \psi} y(\tau)\right] d \tau \\
= & {\left[\frac{(\psi(t)-\psi(\tau))^{\alpha-1}}{\Gamma(\alpha)}\left(\frac{1}{\psi^{\prime}(\tau)} \frac{d}{d \tau}\right)^{n-2} I_{a+}^{n-\alpha, \psi} y(\tau)\right]_{a}^{t} } \\
& +\frac{1}{\Gamma(\alpha-1)} \int_{a}^{t}(\psi(t)-\psi(\tau))^{\alpha-2} \frac{d}{d \tau}\left[\left(\frac{1}{\psi^{\prime}(\tau)} \frac{d}{d \tau}\right)^{n-3} I_{a+}^{n-\alpha, \psi} y(\tau)\right] d \tau \\
= & \frac{1}{\Gamma(\alpha-1)} \int_{a}^{t}(\psi(t)-\psi(\tau))^{\alpha-2} \frac{d}{d \tau}\left[\left(\frac{1}{\psi^{\prime}(\tau)} \frac{d}{d \tau}\right)^{n-3} I_{a+}^{n-\alpha, \psi} y(\tau)\right] d \tau .
\end{aligned}
$$

Repeating this procedure, we arrive to

$$
\begin{aligned}
\star= & {\left[\frac{(\psi(t)-\psi(\tau))^{\alpha-n+2}}{\Gamma(\alpha-n+3)}\left(\frac{1}{\psi^{\prime}(\tau)} \frac{d}{d \tau}\right) I_{a+}^{n-\alpha, \psi} y(\tau)\right]_{a}^{t} } \\
& +\frac{1}{\Gamma(\alpha-n+2)} \int_{a}^{t}(\psi(t)-\psi(\tau))^{\alpha-n+1} \frac{d}{d \tau} l_{a+}^{n-\alpha, \psi} y(\tau) d \tau \\
= & \frac{1}{\Gamma(\alpha-n+2)} \int_{a}^{t}(\psi(t)-\psi(\tau))^{\alpha-n+1} \frac{d}{d \tau} l_{a+}^{n-\alpha, \psi} y(\tau) d \tau \\
= & {\left[\frac{(\psi(t)-\psi(\tau))^{\alpha-n+1}}{\Gamma(\alpha-n+2)} I_{a+}^{n-\alpha, \psi} y(\tau)\right]_{a}^{t}+\frac{1}{\Gamma(\alpha-n+1)} \int_{a}^{t} \psi^{\prime}(\tau)(\psi(t)-\psi(\tau))^{\alpha-n} I_{a+}^{n-\alpha, \psi} y(\tau) d \tau } \\
= & l_{a+}^{\alpha-n+1, \psi} I_{a+}^{n-\alpha, \psi} y(t)=l_{a+}^{1, \psi} y(t) .
\end{aligned}
$$

In conclusion, we prove the desired formula:

$$
I_{a+}^{\alpha, \psi} D_{a+}^{\alpha, \psi} y(t)=\frac{1}{\psi^{\prime}(t)} \frac{d}{d t} I_{a+}^{1, \psi} y(t)=y(t)
$$

\section{Fractional Differential Equations}

This section contains our main results. We prove existence and uniqueness results for the initial value problem of nonlinear FDE involving $\psi$-Caputo fractional derivative ${ }^{C} D_{a+}^{\alpha, \psi} x(t)=f(t, x(t))$, develop the Picard iteration method for solving this problem and for a particular case of FDEs we establish results on the long-term behaviour of solutions

\subsection{Existence and uniqueness of solution}

Consider the problem $(P)$, given by the nonlinear FDE

$$
{ }^{c} D_{a+}^{\alpha, \psi} x(t)=f(t, x(t)), \quad t \in[a, b],
$$

subject to the initial conditions

$$
x(a)=x_{a} \text { and } x_{\psi}^{[k]}(a)=x_{a}^{k}, k=1, \ldots, n-1,
$$

where

1. $0<\alpha \notin \mathbb{N}$ and $n=[\alpha]+1$,

2. $x_{a}$ and $x_{a}^{k}$, for $k=1, \ldots, n-1$, are fixed reals,

3. $x \in C^{n-1}[a, b]$ such that ${ }^{C} D_{a+}^{\alpha, \psi} x$ exists and is continuous in $[a, b]$,

4. $f:[a, b] \times \mathbb{R} \rightarrow \mathbb{R}$ is continuous.

Also, we denote $x_{a}^{0}:=x_{a}$. We first prove an equivalence relation between the fractional Cauchy problem $(P)$ and the Volterra integral equation.

Theorem 2 A function $x \in C^{n-1}[a, b]$ is a solution to problem $(P)$ if and only if $x$ satisfies the following fractional integral equation

$$
x(t)=l_{a+}^{\alpha, \psi} f(t, x(t))+\sum_{k=0}^{n-1} \frac{x_{a}^{k}}{k !}(\psi(t)-\psi(a))^{k} .
$$


Proof This result is a consequence of Theorem 1 . The implication $(P) \Rightarrow(1)$ is clear: applying the operator $l_{a+}^{\alpha, \psi}$ to both sides of the equation ${ }^{C} D_{a+}^{\alpha, \psi} x(t)=f(t, x(t))$ and using the initial conditions, we obtain (1). To prove the converse, we apply the operator ${ }^{C} D_{a+}^{\alpha, \psi}$ to both sides of equation (1) and use the fact that

$$
{ }^{c} D_{a+}^{\alpha, \psi}(\psi(t)-\psi(a))^{k}=0, \quad \forall k \in\{0,1, \ldots, n-1\},
$$

to obtain ${ }^{C} D_{a+}^{\alpha, \psi} x(t)=f(t, x(t))$. Finally, we have to prove that the initial conditions are also met. It is clear that $x(a)=x_{a}$ Also, direct computations lead to

$$
x_{\psi}^{[1]}(t)=\frac{x^{\prime}(t)}{\psi^{\prime}(t)}=\frac{1}{\Gamma(\alpha-1)} \int_{a}^{t} \psi^{\prime}(\tau)(\psi(t)-\psi(\tau))^{\alpha-2} f(\tau, x(\tau)) d \tau+\sum_{k=1}^{n-1} \frac{x_{a}^{k}}{(k-1) !}(\psi(t)-\psi(a))^{k-1},
$$

and so $x_{\psi}^{[1]}(a)=x_{a}^{1}$. Repeating this process, we arrive to

$$
x_{\psi}^{[n-1]}(t)=\frac{\left(x^{[n-2]}(t)\right)^{\prime}}{\psi^{\prime}(t)}=\frac{1}{\Gamma(\alpha-n+1)} \int_{a}^{t} \psi^{\prime}(\tau)(\psi(t)-\psi(\tau))^{\alpha-n} f(\tau, x(\tau)) d \tau+x_{a}^{n-1} .
$$

Since $f(\cdot, x(\cdot))$ is continuous on $[a, b]$, there exists a positive constant $A$ such that

$$
\left|\frac{1}{\Gamma(\alpha-n+1)} \int_{a}^{t} \psi^{\prime}(\tau)(\psi(t)-\psi(\tau))^{\alpha-n} f(\tau, x(\tau)) d \tau\right| \leq A \frac{(\psi(t)-\psi(a))^{\alpha-n+1}}{\Gamma(\alpha-n+2)},
$$

which vanishes at the initial point $t=a$, and thus $x_{\psi}^{[n-1]}(a)=x_{a}^{n-1}$.

Theorem 3 Assume that function $f$ is Lipschitz continuous with respect to the second variable, that is, there exists a positive constant $L$ such that

$$
\left|f\left(t, x_{1}\right)-f\left(t, x_{2}\right)\right| \leq L\left|x_{1}-x_{2}\right|, \quad \forall t \in[a, b] \forall x_{1}, x_{2} \in \mathbb{R} .
$$

Then, there is a constant $h \in \mathbb{R}^{+}$such that there exists a unique solution to problem $(P)$ on the interval $[a, a+h] \subseteq[a, b]$.

Proof Let $h$ be a real satisfying the conditions

$$
L \frac{(\psi(a+h)-\psi(a))^{\alpha}}{\Gamma(\alpha+1)}<1 \quad \text { and } \quad a+h \leq b .
$$

Define the set

$$
U:=\left\{x \in C^{n-1}[a, a+h]:{ }^{C} D_{a+}^{\alpha, \psi} x \in C[a, a+h]\right\},
$$

and the operator $F: U \rightarrow U$ by the rule

$$
F[x](t):=l_{a+}^{\alpha, \psi} f(t, x(t))+\sum_{k=0}^{n-1} \frac{x_{a}^{k}}{k !}(\psi(t)-\psi(a))^{k} .
$$

We first prove that $F$ is well defined, that is, $F(U) \subseteq U$. For that purpose, consider a function $x \in C^{n-1}[a, a+h]$. It is clear that the map $t \mapsto F[x](t)$ is of class $C^{n-1}$. Also,

$$
{ }^{C} D_{a+}^{\alpha, \psi} F[x](t)=\left.{ }^{C} D_{a+}^{\alpha, \psi}\right|_{a+} ^{\alpha, \psi} f(t, x(t))+\sum_{k=0}^{n-1} \frac{x_{a}^{k}}{k !}{ }^{C} D_{a+}^{\alpha, \psi}(\psi(t)-\psi(a))^{k}=f(t, x(t))
$$

is continuous in $[a, a+h]$. Next, we shall show that $F$ is a contraction. Given $x_{1}, x_{2} \in U$, we have

$$
\begin{aligned}
\left\|F\left(x_{1}\right)-F\left(x_{2}\right)\right\| & =\max _{t \in[a, a+h]}\left|F\left[x_{1}\right](t)-F\left[x_{2}\right](t)\right|=\max _{t \in[a, a+h]}\left|I_{a+}^{\alpha, \psi}\left(f\left(t, x_{1}(t)\right)-f\left(t, x_{2}(t)\right)\right)\right| \\
& \leq L \frac{(\psi(a+h)-\psi(a))^{\alpha}}{\Gamma(\alpha+1)}\left\|x_{1}-x_{2}\right\|,
\end{aligned}
$$

which proves that $F$ is a contraction. By the Banach fixed point theorem, we get the result of the theorem.

Next, we prove the existence of a solution to the fractional Cauchy problem $(P)$ using on the Schaefer fixed point theorem.

Theorem 4 Assume that function $f$ is continuous and there exist two positive constants $k_{0}$ and $k_{1}$ such that

$$
|f(t, x)| \leq k_{0}+k_{1}|x|, \quad \forall t \in[a, b] \forall x \in \mathbb{R} .
$$

Then, there exists a constant $h>0$ such that problem $(P)$ has at least one solution defined on the interval $[a, a+h] \subseteq[a, b]$. 
Proof Let $h>0$ be such that $a+h \leq b$ and

$$
1-\frac{k_{1}}{\Gamma(\alpha+1)}(\psi(a+h)-\psi(a))^{\alpha}>0
$$

Consider the set $U$ and the operator $F: U \rightarrow U$ defined by (3) and (4), respectively. We shall divide the proof in four steps.

Claim I: $F$ is continuous.

Let $\left(x_{n}\right)$ be a sequence converging to $x$ in $U$. Then,

$$
\begin{aligned}
\left\|F\left(x_{n}\right)-F(x)\right\| & =\max _{t \in[a, a+h]}\left|F\left[x_{n}\right](t)-F[x](t)\right|=\max _{t \in[a, a+h]}\left|I_{a+}^{\alpha, \psi}\left(f\left(t, x_{n}(t)\right)-f(t, x(t))\right)\right| \\
& \leq\left\|f\left(\cdot, x_{n}(\cdot)\right)-f(\cdot, x(\cdot))\right\| \frac{(\psi(a+h)-\psi(a))^{\alpha}}{\Gamma(\alpha+1)} .
\end{aligned}
$$

Since $f$ is a continuous function, we have

$$
F\left(x_{n}\right)-F(x) \rightarrow 0 \text { as } n \rightarrow \infty .
$$

Claim II: $F$ maps bounded sets into bounded sets in $U$.

We prove that, for all $r>0$, there exists some $r^{\prime}>0$ such that

$$
\forall x \in A_{r}:=\{x \in U:\|x\| \leq r\}:\|F(x)\| \leq r^{\prime} .
$$

Indeed, given $x \in A_{r}$, and using the relation

$$
|f(t, x(t))| \leq k_{0}+k_{1}\|x\| \leq k_{0}+k_{1} r, \quad \forall t \in[a, a+h],
$$

we have

$$
\|F(x)\| \leq \frac{k_{0}+k_{1} r}{\Gamma(\alpha+1)}(\psi(a+h)-\psi(a))^{\alpha}+\sum_{k=0}^{n-1} \frac{\left|x_{a}^{k}\right|}{k !}(\psi(a+h)-\psi(a))^{k}:=r^{\prime},
$$

which is independent of $t$ and $x$, and so $F$ is uniformly bounded.

Claim III: $F$ maps bounded sets into equicontinuous sets in $U$.

Let $t_{1}, t_{2} \in[a, a+h]$ with $t_{1}<t_{2}, A_{r}$ defined as in Claim II, and set the signum function

$$
\operatorname{sgn}(\alpha):=\left\{\begin{aligned}
1 & \text { if } \alpha>1 \\
-1 & \text { if } \alpha \in(0,1)
\end{aligned}\right.
$$

Then, for all $x \in A_{r}$ :

$$
\begin{aligned}
\mid F[x] & \left(t_{2}\right)-F[x]\left(t_{1}\right) \mid \\
\leq & ||_{a+}^{\alpha, \psi}\left(f\left(t_{2}, x\left(t_{2}\right)\right)-f\left(t_{1}, x\left(t_{1}\right)\right)\right) \mid+\sum_{k=0}^{n-1} \frac{\left|x_{a}^{k}\right|}{k !}\left[\left(\psi\left(t_{2}\right)-\psi(a)\right)^{k}-\left(\psi\left(t_{1}\right)-\psi(a)\right)^{k}\right] \\
= & \frac{1}{\Gamma(\alpha)}\left|\int_{a}^{t_{2}} \psi^{\prime}(\tau)\left(\psi\left(t_{2}\right)-\psi(\tau)\right)^{\alpha-1} f(\tau, x(\tau)) d \tau-\int_{a}^{t_{1}} \psi^{\prime}(\tau)\left(\psi\left(t_{1}\right)-\psi(\tau)\right)^{\alpha-1} f(\tau, x(\tau)) d \tau\right| \\
& +\sum_{k=0}^{n-1} \frac{\left|x_{a}^{k}\right|}{k !}\left[\left(\psi\left(t_{2}\right)-\psi(a)\right)^{k}-\left(\psi\left(t_{1}\right)-\psi(a)\right)^{k}\right] \\
\leq & \frac{k_{0}+k_{1} r}{\Gamma(\alpha)}\left[\int_{a}^{t_{1}} \operatorname{sgn}(\alpha) \psi^{\prime}(\tau)\left[\left(\psi\left(t_{2}\right)-\psi(\tau)\right)^{\alpha-1}-\left(\psi\left(t_{1}\right)-\psi(\tau)\right)^{\alpha-1}\right] d \tau\right. \\
& \left.+\int_{t_{1}}^{t_{2}} \psi^{\prime}(\tau)\left(\psi\left(t_{2}\right)-\psi(\tau)\right)^{\alpha-1} d \tau\right]+\sum_{k=0}^{n-1} \frac{\left|x_{a}^{k}\right|}{k !}\left[\left(\psi\left(t_{2}\right)-\psi(a)\right)^{k}-\left(\psi\left(t_{1}\right)-\psi(a)\right)^{k}\right] \\
\leq & \frac{k_{0}+k_{1} r}{\Gamma(\alpha+1)}\left[\operatorname{sgn}(\alpha)\left[\left(\psi\left(t_{2}\right)-\psi(a)\right)^{\alpha}-\left(\psi\left(t_{2}\right)-\psi\left(t_{1}\right)\right)^{\alpha}-\left(\psi\left(t_{1}\right)-\psi(a)\right)^{\alpha}\right]+\left(\psi\left(t_{2}\right)-\psi\left(t_{1}\right)\right)^{\alpha}\right] \\
& +\sum_{k=0}^{n-1} \frac{\left|x_{a}^{k}\right|}{k !}\left[\left(\psi\left(t_{2}\right)-\psi(a)\right)^{k}-\left(\psi\left(t_{1}\right)-\psi(a)\right)^{k}\right] .
\end{aligned}
$$

Since the right-hand side of the above inequality converges to zero as $t_{2} \rightarrow t_{1}$, we have that $F[x]\left(t_{2}\right) \rightarrow F[x]\left(t_{1}\right)$. As a consequence of Claims I-III together with the Arzelá-Ascoli Theorem, we conclude that $F$ is completely continuous.

Claim IV: To conclude the proof, we show that the set

$$
T:=\{x \in U: x=\lambda F(x) \text { for some } \lambda \in(0,1)\}
$$


is bounded. Let $x \in T$ and $\lambda \in(0,1)$ be such that $x=\lambda F(x)$. For all $t \in[a, a+h]$, we have

$$
|F[x](t)| \leq \frac{k_{0}+k_{1}\|x\|}{\Gamma(\alpha+1)}(\psi(t)-\psi(a))^{\alpha}+\sum_{k=0}^{n-1} \frac{\left|x_{a}^{k}\right|}{k !}(\psi(t)-\psi(a))^{k},
$$

and so

$$
\begin{gathered}
\|x\|<\|F(x)\| \leq \frac{k_{0}+k_{1}\|x\|}{\Gamma(\alpha+1)}(\psi(a+h)-\psi(a))^{\alpha}+\sum_{k=0}^{n-1} \frac{\left|x_{a}^{k}\right|}{k !}(\psi(a+h)-\psi(a))^{k}, \\
\Leftrightarrow\|x\| \leq \frac{\frac{k_{0}}{\Gamma(\alpha+1)}(\psi(a+h)-\psi(a))^{\alpha}+\sum_{k=0}^{n-1} \frac{\left|x_{a}^{k}\right|}{k !}(\psi(a+h)-\psi(a))^{k}}{1-\frac{k_{1}}{\Gamma(\alpha+1)}(\psi(a+h)-\psi(a))^{\alpha}},
\end{gathered}
$$

which proves that $T$ is bounded. By the Schaefer fixed point theorem, $F$ has a fixed point.

Corollary 1 Assume that function $f$ is continuous and bounded. Then, there is at least one solution to problem $(P)$, defined on some interval $[a, a+h]$.

\subsection{Picard iteration}

Let us recall that, by Theorem $2, x$ is a solution of problem $(P)$ if and only if $x$ is a solution of the equation

$$
x(t)=l_{a+}^{\alpha, \psi} f(t, x(t))+\sum_{k=0}^{n-1} \frac{x_{a}^{k}}{k !}(\psi(t)-\psi(a))^{k} .
$$

Consider the sequence of functions $\left(x_{m}\right)_{m=0}^{\infty}$, defined recursively through

$$
\left\{\begin{array}{l}
x_{0}(t)=\sum_{k=0}^{n-1} \frac{x_{a}^{k}}{k !}(\psi(t)-\psi(a))^{k} \\
x_{m+1}(t)=l_{a+}^{\alpha, \psi} f\left(t, x_{m}(t)\right)+\sum_{k=0}^{n-1} \frac{x_{a}^{k}}{k !}(\psi(t)-\psi(a))^{k}, \quad m=0,1,2, \ldots
\end{array}\right.
$$

Under the assumptions of Theorem 3 , we shall prove that $\left(x_{m}\right)_{m=0}^{\infty}$ converges uniformly on the interval $[a, b]$ to a function $x$ that verifies equation (5). Set

$$
M:=\max _{t \in[a, b]}\left|f\left(t, x_{0}(t)\right)\right| .
$$

We prove, by induction, that for all $m \in \mathbb{N} \cup\{0\}$, the following inequality holds:

$$
\left|x_{m+1}(t)-x_{m}(t)\right| \leq M \frac{L^{m}}{\Gamma((m+1) \alpha+1)}(\psi(t)-\psi(a))^{(m+1) \alpha} .
$$

First, we have that

$$
\left|x_{1}(t)-x_{0}(t)\right|=\left|l_{a+}^{\alpha, \psi} f\left(t, x_{0}(t)\right)\right| \leq M I_{a+}^{\alpha, \psi} 1=M \frac{(\psi(t)-\psi(a))^{\alpha}}{\Gamma(\alpha+1)} .
$$

On the other hand,

$$
\begin{aligned}
\left|x_{m+2}(t)-x_{m+1}(t)\right| \leq & l_{a+}^{\alpha, \psi}\left|f\left(t, x_{m+1}(t)\right)-f\left(t, x_{m}(t)\right)\right| \leq L l_{a+}^{\alpha, \psi}\left|x_{m+1}(t)-x_{m}(t)\right| \\
\leq & M \frac{L^{m+1}}{\Gamma((m+1) \alpha+1)} l_{a+}^{\alpha, \psi}(\psi(t)-\psi(a))^{(m+1) \alpha} \\
= & M \frac{L^{m+1}}{\Gamma((m+1) \alpha+1) \Gamma(\alpha)}(\psi(t)-\psi(a))^{\alpha-1} \\
& \times \int_{a}^{t} \psi^{\prime}(\tau)\left(1-\frac{\psi(\tau)-\psi(a)}{\psi(t)-\psi(a)}\right)^{\alpha-1}(\psi(\tau)-\psi(a))^{(m+1) \alpha} d \tau .
\end{aligned}
$$

Proceeding with the change of variables

$$
u=\frac{\psi(\tau)-\psi(a)}{\psi(t)-\psi(a)}
$$

and with the help of the Beta function $B(\cdot, \cdot)$, we arrive to

$$
\begin{aligned}
\left|x_{m+2}(t)-x_{m+1}(t)\right| & \leq M \frac{L^{m+1}}{\Gamma((m+1) \alpha+1) \Gamma(\alpha)}(\psi(t)-\psi(a))^{(m+2) \alpha} \int_{0}^{1}(1-u)^{\alpha-1} u^{(m+1) \alpha} d u \\
& =M \frac{L^{m+1}}{\Gamma((m+1) \alpha+1) \Gamma(\alpha)}(\psi(t)-\psi(a))^{(m+2) \alpha} B(\alpha,(m+1) \alpha+1) \\
& =M \frac{L^{m+1}}{\Gamma((m+2) \alpha+1)}(\psi(t)-\psi(a))^{(m+2) \alpha} .
\end{aligned}
$$


Now, we prove that the series

$$
\sum_{m=0}^{\infty} M \frac{L^{m}}{\Gamma((m+1) \alpha+1)}(\psi(b)-\psi(a))^{(m+1) \alpha}
$$

is convergent. Applying the ratio test, we get

$$
\lim _{m \rightarrow \infty} \frac{M \frac{L^{m+1}}{\Gamma((m+2) \alpha+1)}(\psi(b)-\psi(a))^{(m+2) \alpha}}{M \frac{L^{m}}{\Gamma((m+1) \alpha+1)}(\psi(b)-\psi(a))^{(m+1) \alpha}}=L(\psi(b)-\psi(a))^{\alpha} \lim _{m \rightarrow \infty} \frac{\Gamma((m+1) \alpha+1)}{\Gamma((m+1) \alpha+1+\alpha)}=0,
$$

since (see Eq. (1) in [24])

$$
\frac{\Gamma((m+1) \alpha+1)}{\Gamma((m+1) \alpha+1+\alpha)}=\frac{1}{((m+1) \alpha+1)^{\alpha}}\left(1-\frac{\alpha(\alpha-1)}{2((m+1) \alpha+1)}+O\left(((m+1) \alpha+1)^{-2}\right)\right) .
$$

Attending that

$$
\left|x_{m+1}(t)-x_{m}(t)\right| \leq M \frac{L^{m}}{\Gamma((m+1) \alpha+1)}(\psi(b)-\psi(a))^{(m+1) \alpha},
$$

for all $t \in[a, b]$, by the Weierstrass M-test [26], we conclude that the series

$$
\sum_{m=0}^{\infty}\left(x_{m+1}(t)-x_{m}(t)\right)+\sum_{k=0}^{n-1} \frac{x_{a}^{k}}{k !}(\psi(t)-\psi(a))^{k}
$$

converges uniformly in $[a, b]$, and let $x$ be its limit. Observe that, for all $/ \in \mathbb{N}$,

$$
\sum_{m=0}^{1-1}\left(x_{m+1}(t)-x_{m}(t)\right)+\sum_{k=0}^{n-1} \frac{x_{a}^{k}}{k !}(\psi(t)-\psi(a))^{k}=x_{l}(t)
$$

and since

$$
\left|f\left(t, x_{l}(t)\right)-f(t, x(t))\right| \leq L\left|x_{l}(t)-x(t)\right|,
$$

we get that $f\left(\cdot, x_{l}(\cdot)\right)$ converges uniformly to $f(\cdot, x(\cdot))$ in $[a, b]$, as $I \rightarrow \infty$. Since

$$
x_{m+1}(t)=l_{a+}^{\alpha, \psi} f\left(t, x_{m}(t)\right)+\sum_{k=0}^{n-1} \frac{x_{a}^{k}}{k !}(\psi(t)-\psi(a))^{k}, \quad m=0,1,2, \ldots,
$$

letting $m \rightarrow \infty$, we deduce

$$
x(t)=I_{a+}^{\alpha, \psi} f(t, x(t))+\sum_{k=0}^{n-1} \frac{x_{a}^{k}}{k !}(\psi(t)-\psi(a))^{k},
$$

that is, $x$ is a solution to problem $(P)$. Also, we have the upper bound

$$
\begin{aligned}
\left|x(t)-\sum_{k=0}^{n-1} \frac{x_{a}^{k}}{k !}(\psi(t)-\psi(a))^{k}\right| & \leq \sum_{m=0}^{\infty}\left|x_{m+1}(t)-x_{m}(t)\right| \\
& \leq \sum_{m=0}^{\infty} M \frac{L^{m}}{\Gamma((m+1) \alpha+1)}(\psi(t)-\psi(a))^{(m+1) \alpha} \\
& \leq \frac{M}{L} \sum_{m=1}^{\infty} \frac{\left(L(\psi(t)-\psi(a))^{\alpha}\right)^{m}}{\Gamma(m \alpha+1)} \\
& =\frac{M}{L}\left(E_{\alpha}\left(L(\psi(t)-\psi(a))^{\alpha}\right)-1\right),
\end{aligned}
$$

where $E_{\alpha}$ is the Mittag-Leffler function:

$$
E_{\alpha}(z):=\sum_{k=0}^{\infty} \frac{z^{k}}{\Gamma(k \alpha+1)}
$$

Example 1 Consider the system

$$
\left\{\begin{array}{l}
{ }^{C} D_{0+}^{1.5, \psi} x(t)=x(t) \\
x(0)=1, \quad x_{\psi}^{[1]}(0)=0
\end{array}\right.
$$

The solution of this problem is the function (see Lemma 2 in [5]).

$$
x(t)=E_{\alpha}\left((\psi(t)-\psi(0))^{1.5}\right) .
$$


For this case, the Picard iterative process is described as

$$
\left\{\begin{array}{l}
x_{0}(t)=1 \\
x_{m+1}(t)=1+I_{0+}^{1.5, \psi} x_{m}(t), \quad m=0,1,2, \ldots
\end{array}\right.
$$

In Figure 1, we present the results for two different kernels: (a) $\psi(t)=t$, (b) $\psi(t)=t^{2}$. In both cases, we present the plot of the exact solution $x$ and three approximations of it, by considering $x_{1}, x_{3}$ and $x_{5}$, in the Picard iterations.

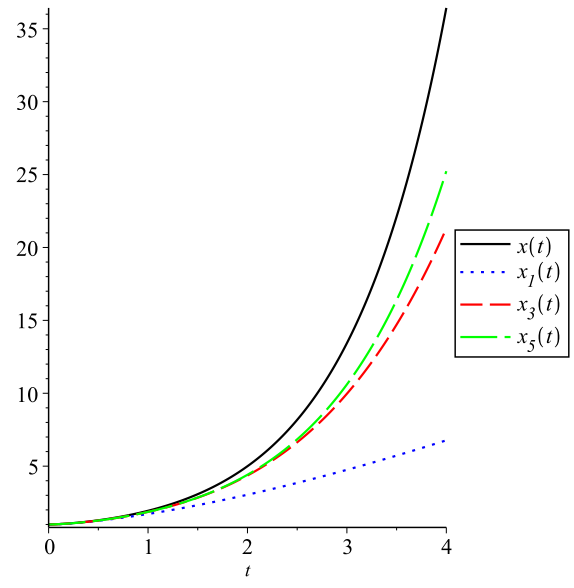

(a) For $\psi(t)=t$

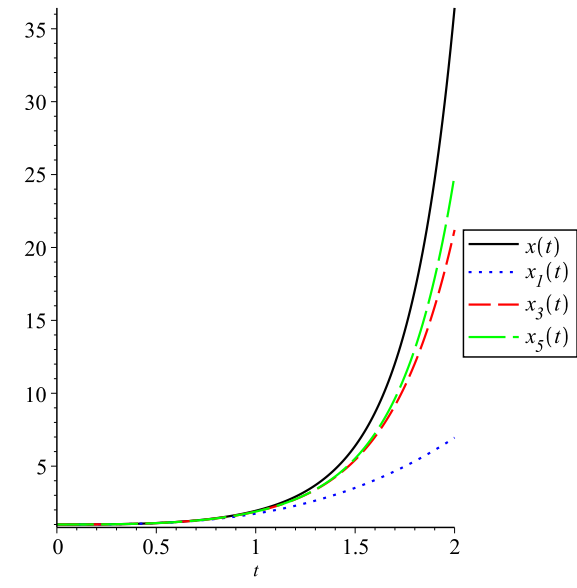

(b) For $\psi(t)=t^{2}$

Figure 1. The Picard iterations with respect to the two different kernels.

\subsection{The long-term behaviour of solutions}

Consider the following initial value problem

$$
{ }^{c} D_{a+}^{\alpha, \psi} x(t)=f(t, x(t)), \quad x(a)=x_{a}
$$

where $0<\alpha<1, x_{a} \in \mathbb{R}$. We assume that $\psi:[a, \infty) \rightarrow \mathbb{R}$ is an increasing and unbounded function such that $\psi^{\prime}(x) \neq 0$ for all $t \geq a$. We are interested in the behaviour of the solutions of (6) when $t \rightarrow \infty$. Therefore, we have to extend previously presented results on the existence and uniqueness of solutions.

Theorem 5 Assume that function $f:[a, b] \times \mathbb{R} \rightarrow \mathbb{R}$ is continuous and Lipschitz with respect to the second variable, that is, condition (2) holds. Then, there exists a unique solution $x \in C[a, b]$ to problem (6).

Proof Let $t_{1} \in \mathbb{R}$ be such that $a<t_{1}<b$ and

$$
L \frac{\left(\psi\left(t_{1}\right)-\psi(a)\right)^{\alpha}}{\Gamma(\alpha+1)}<1 .
$$

Then, using the same method applied in the proof of Theorem 3 , we can show that there exists a unique solution $x^{1} \in C\left[a, t_{1}\right]$ to problem (6). This solution can be found applying the Picard iteration, presented in Section 3.2. Now observe that, by Theorem 2 , we can write a solution to (6) in the form:

$$
x(t)=x_{a}+\frac{1}{\Gamma(\alpha)} \int_{a}^{t_{1}} \psi^{\prime}(\tau)(\psi(t)-\psi(\tau))^{\alpha-1} f(\tau, x(\tau)) d \tau+\frac{1}{\Gamma(\alpha)} \int_{t_{1}}^{t} \psi^{\prime}(\tau)(\psi(t)-\psi(\tau))^{\alpha-1} x(\tau) d \tau .
$$

Given that over the interval $\left[a, t_{1}\right]$ equation (6) possesses a unique solution, we can rewrite ( 7 ) as follows

$$
x(t)=x_{0}(t)+\frac{1}{\Gamma(\alpha)} \int_{t_{1}}^{t} \psi^{\prime}(\tau)(\psi(t)-\psi(\tau))^{\alpha-1} x(\tau) d \tau
$$

where

$$
x_{0}(t)=x_{a}+\frac{1}{\Gamma(\alpha)} \int_{a}^{t_{1}} \psi^{\prime}(\tau)(\psi(t)-\psi(\tau))^{\alpha-1} f(\tau, x(\tau)) d \tau
$$

is a known function. Now, let choose $t_{2}=t_{1}+h_{1}$ with $h_{1}>0$, such that $t_{2}<b$ and

$$
L \frac{\left(\psi\left(t_{2}\right)-\psi\left(t_{1}\right)\right)^{\alpha}}{\Gamma(\alpha+1)}<1 .
$$


By the same arguments as in the proof of Theorem 3 , we can show that there exists a unique solution $x^{2} \in C\left[t_{1}, t_{2}\right]$ to equation

(6). Repeating the previous reasoning, choosing $t_{k}=t_{k-1}+h_{k-1}$ with $h_{k-1}>0$ such that $t_{k}<b$ and

$$
L \frac{\left(\psi\left(t_{k}\right)-\psi\left(t_{k-1}\right)\right)^{\alpha}}{\Gamma(\alpha+1)}<1
$$

we can show that problem (6) possesses a unique solution $x^{k} \in C\left[t_{k-1}, t_{k}\right]$ on each interval $\left[t_{k-1}, t_{k}\right], k=1, \ldots, l$, where $a=t_{0}<t_{1}<\ldots<t_{l}=b$. This shows that there exists a unique piecewise continuous solution to problem (6) on the interval $[a, b]$. However, since $\psi$ is continuous, we have

$$
\lim _{\varepsilon \rightarrow 0^{+}} \int_{t}^{t+\varepsilon} \psi^{\prime}(\tau)(\psi(t)-\psi(\tau))^{\alpha-1} d \tau=0
$$

Therefore, the obtained solution is continuous on $[a, b]$

Corollary 2 Assume that function $f:[a, \infty) \times \mathbb{R} \rightarrow \mathbb{R}$ is continuous and Lipschitz with respect to the second variable, that is, condition (2) holds for all $t \in[a, \infty)$ and all $x_{1}, x_{2} \in \mathbb{R}$. Then, there exists a unique solution $x \in C[a, \infty)$ to problem (6).

Proof Note that, under assumptions of Corollary 2, we can apply Theorem 5. Since we can take $b>a$ arbitrary large, it follows that there exists a uniquely defined function $x \in C[a, \infty)$ solving problem (6).

Observe that, if $f(t, 0)=0$ for all $t \geq a$, and $x_{a}=0$, then the function $x(t)=0$ is a solution to initial value problem (6). Below we prove conditions ensuring that a solution to (6), with $x_{a}>0$, converges to zero as $t \rightarrow \infty$.

Theorem 6 Consider problem (6) with $x(a)=x_{a}>0$ and function $f:[a, \infty) \times\left[0, x_{a}\right] \rightarrow(-\infty, 0]$ being continuous and Lipschitz with respect to the second variable, that is, condition (2) holds for all $t \in[a, \infty)$ and all $x_{1}, x_{2} \in\left[0, x_{a}\right]$. Moreover, assume that $f(t, 0)=0$ for all $t$. Then, the unique solution to (6) exists on $[a, \infty)$ and satisfies $0 \leq x(t) \leq x_{a}$ for all $t \geq a$.

Proof By Theorem 2, a solution to (6) satisfies the following

$$
x(t)=x_{a}+\frac{1}{\Gamma(\alpha)} \int_{a}^{t} \psi^{\prime}(\tau)(\psi(t)-\psi(\tau))^{\alpha-1} f(\tau, x(\tau)) d \tau
$$

Define

$$
\tilde{f}(t, y)= \begin{cases}f\left(t, x_{a}\right) & \text { if } y>x_{a} \\ f(t, y) & \text { if } 0 \leq y \leq x_{a} \\ 0 & \text { if } y<0\end{cases}
$$

and in this way extend the domain of $f$ to $[a, \infty) \times \mathbb{R}$. The extended function $\tilde{f}$ satisfies assumptions of Corollary 2 . Therefore, problem (6), with function (9), has a unique continuous solution $x$. Now, we prove that this solution satisfies inequality $0 \leq x(t) \leq x_{a}$ for all $t \geq a$. Suppose that $x$ changes the sign, that is

$$
\begin{cases}x(t)>0 & \text { for } a \leq t<t_{1} \\ x(t)=0 & \text { for } t=t_{1} \\ x(t)<0 & \text { for } t_{1}<t \leq t_{2}\end{cases}
$$

Thus, for the extended $\tilde{f}$ we have

$$
\begin{cases}\tilde{f}(t, x(t)) \leq 0 & \text { for } a \leq t \leq t_{1} \\ \tilde{f}(t, x(t))=0 & \text { for } t_{1}<t \leq t_{2}\end{cases}
$$

Combining (10) with (8) yields

$$
\begin{aligned}
x\left(t_{2}\right) & =x_{a}+\frac{1}{\Gamma(\alpha)} \int_{a}^{t_{2}} \psi^{\prime}(\tau)\left(\psi\left(t_{2}\right)-\psi(\tau)\right)^{\alpha-1} \tilde{f}(\tau, x(\tau)) d \tau \\
& =x_{a}+\frac{1}{\Gamma(\alpha)} \int_{a}^{t_{1}} \psi^{\prime}(\tau)\left(\psi\left(t_{2}\right)-\psi(\tau)\right)^{\alpha-1} \tilde{f}(\tau, x(\tau)) d \tau \\
& +\frac{1}{\Gamma(\alpha)} \int_{t_{1}}^{t_{2}} \psi^{\prime}(\tau)\left(\psi\left(t_{2}\right)-\psi(\tau)\right)^{\alpha-1} \tilde{f}(\tau, x(\tau)) d \tau \\
& =x_{a}+\frac{1}{\Gamma(\alpha)} \int_{a}^{t_{1}} \psi^{\prime}(\tau)\left(\psi\left(t_{2}\right)-\psi(\tau)\right)^{\alpha-1} \tilde{f}(\tau, x(\tau)) d \tau \\
& \geq x_{a}+\frac{1}{\Gamma(\alpha)} \int_{a}^{t_{1}} \psi^{\prime}(\tau)\left(\psi\left(t_{1}\right)-\psi(\tau)\right)^{\alpha-1} \tilde{f}(\tau, x(\tau)) d \tau \\
& =x\left(t_{1}\right)=0,
\end{aligned}
$$


because $\psi$ is an increasing function. But $x\left(t_{2}\right)<0$, a contradiction. Hence, $x(t) \geq 0$ for all $t \geq a$. Now, observe that, by definition, $\tilde{f}(t, x) \leq 0$ for all $t$ and $x$. Therefore, by (8), $x(t) \leq x_{a}$. We have shown that $0 \leq x(t) \leq x_{a}$ for all $t$. This means that $(t, x(t))$ is in the original domain of $f$ and we conclude that $x$ is the unique solution to originally considered problem.

Theorem 7 Consider problem (6) with $x(a)=x_{a}>0$ and function $f:[a, \infty) \times\left[0, x_{a}\right] \rightarrow(-\infty, 0]$ being continuous and Lipschitz with respect to the second variable. Moreover, assume that $f(t, 0)=0$ for all $t$, and that for all $b>0$ and all continuous functions $y:[a, \infty) \rightarrow\left[b, x_{a}\right]$ the following

$$
\lim _{t \rightarrow \infty} l_{a+}^{\alpha, \psi} f(t, y(t))=-\infty
$$

holds. Then, the unique solution to (6) satisfies $\lim _{t \rightarrow \infty} x(t)=0$.

Proof Firstly, observe that by Theorem 6 there exists a unique solution to problem (6), say $x$, such that $0 \leq x(t) \leq x_{a}$ for all $t \geq a$. Therefore, $\lim _{t \rightarrow \infty} x(t) \geq 0$. Suppose that $\lim _{t \rightarrow \infty} x(t)=g>0$ and define

$$
y(t)= \begin{cases}x(t) & \text { for } t>t_{0} \\ x\left(t_{0}\right) & \text { for } t \leq t_{0}\end{cases}
$$

where $t_{0}$ is such that $x(t) \geq g / 2$ for all $t \geq t_{0}$. Clearly, $y:[a, \infty) \rightarrow\left[g / 2, x_{a}\right]$ is a continuous function. Applying Theorem 2 for $t>t_{0}$, we get

$$
\begin{aligned}
x(t)= & x_{a}+\frac{1}{\Gamma(\alpha)} \int_{a}^{t} \psi^{\prime}(\tau)(\psi(t)-\psi(\tau))^{\alpha-1} f(\tau, x(\tau)) d \tau \\
= & x_{a}+\frac{1}{\Gamma(\alpha)} \int_{a}^{t} \psi^{\prime}(\tau)(\psi(t)-\psi(\tau))^{\alpha-1}[f(\tau, x(\tau))-f(\tau, y(\tau))] d \tau \\
& +\frac{1}{\Gamma(\alpha)} \int_{a}^{t} \psi^{\prime}(\tau)(\psi(t)-\psi(\tau))^{\alpha-1} f(\tau, y(\tau)) d \tau
\end{aligned}
$$

Observe that the second term in (13) is bounded. Indeed, we have

$$
\begin{aligned}
& \left|\int_{a}^{t} \psi^{\prime}(\tau)(\psi(t)-\psi(\tau))^{\alpha-1}[f(\tau, x(\tau))-f(\tau, y(\tau))] d \tau\right| \\
& =\left|\int_{a}^{t_{0}} \psi^{\prime}(\tau)(\psi(t)-\psi(\tau))^{\alpha-1}[f(\tau, x(\tau))-f(\tau, y(\tau))] d \tau\right| \\
& \leq\left|\int_{a}^{t_{0}} \psi^{\prime}(\tau)(\psi(t)-\psi(\tau))^{\alpha-1} f(\tau, x(\tau)) d \tau\right|+\left|\int_{a}^{t_{0}} \psi^{\prime}(\tau)(\psi(t)-\psi(\tau))^{\alpha-1} f\left(\tau, x\left(t_{0}\right)\right) d \tau\right| \\
& \leq 2 \sup _{\tau \in\left[a, t_{0}\right], z \in\left[0, x_{a}\right]}|f(\tau, z)| \frac{1}{\alpha}\left[(\psi(t)-\psi(a))^{\alpha}-\left(\psi(t)-\psi\left(t_{0}\right)\right)^{\alpha}\right]
\end{aligned}
$$

As $\psi$ is increasing, the last expression is a positive and decreasing function of $t$ and hence bounded. Now, letting $t \rightarrow \infty$ in (13) we obtain

$$
\begin{aligned}
\lim _{t \rightarrow \infty} x(t) & =\lim _{t \rightarrow \infty}\left(x_{a}+\frac{1}{\Gamma(\alpha)} \int_{a}^{t} \psi^{\prime}(\tau)(\psi(t)-\psi(\tau))^{\alpha-1}[f(\tau, x(\tau))-f(\tau, y(\tau))] d \tau\right. \\
& \left.+\frac{1}{\Gamma(\alpha)} \int_{a}^{t} \psi^{\prime}(\tau)(\psi(t)-\psi(\tau))^{\alpha-1} f(\tau, y(\tau)) d \tau\right)
\end{aligned}
$$

and, by assumption (11), we have $\lim _{t \rightarrow \infty} x(t)=-\infty$, which contradicts to $x(t) \geq 0$. Hence, $\lim _{t \rightarrow \infty} x(t)=0$.

Example 2 Consider the following initial value problem

$$
{ }^{c} D_{a+}^{\alpha, \psi} x(t)=-\lambda x(t), \quad x(a)=x_{a}>0, \quad \lambda>0 .
$$

Note that assumptions of Theorem 7 are satisfied. In fact, since $\psi$ is an increasing and unbounded function, for all $b>0$ and all continuous functions $y:[a, \infty) \rightarrow\left[b, x_{a}\right]$, we have the following:

$$
\lim _{t \rightarrow \infty} l_{a+}^{\alpha, \psi}(-\lambda y(t)) \leq-\lambda b \lim _{t \rightarrow \infty} \frac{(\psi(t)-\psi(a))^{\alpha}}{\Gamma(\alpha+1)}=-\infty .
$$

Therefore, there exists a unique solution $x$ to (14), and such solution satisfies the condition $\lim _{t \rightarrow \infty} x(t)=0$. Figure 2 presents the solutions to problem (14) for different values of $\alpha$, with $\lambda=2$. In plot (a), the kernel is the function $\psi(t)=2^{t}$, and in plot (b), the kernel is $\psi(t)=t$. We remark that case (b) corresponds to the (usual) Caputo fractional derivative, with $\alpha=1$ being the classical derivative. Figure shows similarities between corresponding results with the $\psi$-Caputo derivative and the Caputo derivative. However, $\psi$-Caputo derivative provides, besides the order, any number of free parameters to better calibrate a system. This is a major advantage of the $\psi$-Caputo derivative over the classical fractional derivatives. 


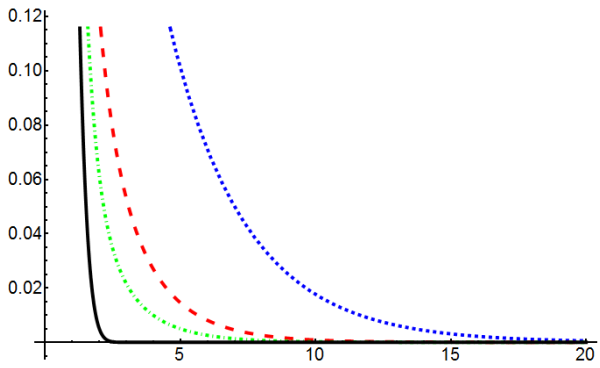

$\ldots=0.5 \ldots=0.8 \ldots \alpha=0.9-\alpha=1$

(a) For $\psi(t)=2^{t}$.

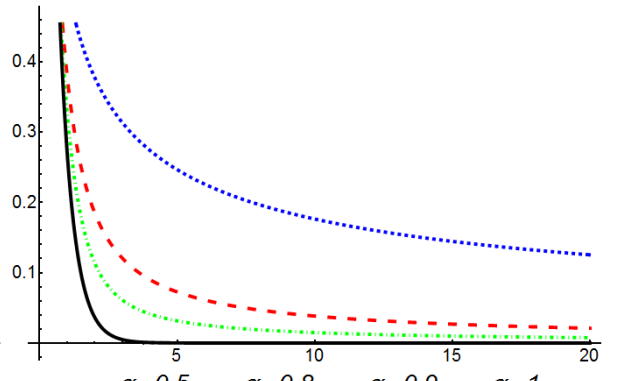

(b) For $\psi(t)=t$.

Figure 2. Solutions to equation (14) with $\lambda=2$, different values of $\alpha$ and for two kernels.

\section{Applications to real-world problems}

In this section, we consider applications of FDE's, with $\psi$-Caputo fractional derivatives, to the world population growth and the gross domestic product (GDP) of some countries. The goal is to determine the order $\alpha$ and the kernel $\psi$ in such a way that solutions to FDE's fit with given real data. Obtained in this way a fractional model, we then compared it with the classical one (that is, when the order is $\alpha=1$ and the kernel is $\psi(t)=t$ ). The least squares fitting technique (routine lsqcurvefit from the Matlab Optimization Toolbox [20]) will be used to provide a solution to the problem of finding the values of the unknown parameter $A$ of the theoretical model $x=F(A, t)$. Given a certain data consisting of $N$ points $\left(\tilde{t}_{i}, \tilde{x}_{i}\right)_{i=1, \ldots, N}$, the goal is to minimize the sum of squared residuals, a residual being the difference between an observed value and the fitted value provided by the model:

$$
\min E_{a b s}=\min \sum_{i=1}^{N}\left[\tilde{x}_{i}-F\left(A, \tilde{t}_{i}\right)\right]^{2} .
$$

Since $\psi$ is a increasing function, we impose the constraint $\psi\left(\tilde{t}_{i}\right)<\psi\left(\tilde{t}_{i+1}\right)$, for all $i$. In each of the two applications, we will find the optimal parameters for fractional models, that is, the order $\alpha$ and the values of the kernel in the data $\psi\left(\tilde{t}_{i}\right)$, for $i=1, \ldots, N$. To compare the efficiency of the procedure, we present in each case the relative errors

$$
E_{r e l}=\frac{\sum_{i=1}^{N}\left[\tilde{x}_{i}-F\left(A, \tilde{t}_{i}\right)\right]^{2}}{\sum_{i=1}^{N}\left(\tilde{x}_{i}\right)^{2}} .
$$

In our calculations, we use the data available from [23], consisting of 28 values, from the year 1960 until 2014, measured every two years. Then, using the best estimative of the parameters, we determine an approximation of the data with respect to the odd years. To estimate the values of $\psi$ in the odd years we do the following procedure: for each $i, \psi\left(t_{2 i-1}\right)=\left(\psi\left(t_{2 i-2}\right)+\psi\left(t_{2 i}\right)\right) / 2$, excluding 2015, where $\psi(2015)=\psi(2014)$

\subsection{World population growth}

In 1798, the British economist Thomas Malthus published a book entitled An Essay on the Principle of Population, where several issues were considered regarding the growth of the human population. Malthus purposed a theoretical model, were the human population exhibits exponential growth, being described by the linear differential equation $N^{\prime}(t)=\lambda N(t)$, where $\lambda$ is the net growth rate (birth rate minus death rate in population) and it is assumed to be constant in time. The solution of this differential equation is the exponential function

$$
N(t)=N_{0} \exp (\lambda t)
$$

where $N_{0}$ is the population at time $t=0$. This model does not take into account the natural constraints of the system, like the food and space availability, the competition between species, pollution, etc. Thus, more complex differential equations may be purposed to deal with such problems regarding real data (e.g. Verhulst and augmented logistic models). However, the Malthusian type model with the $\psi$-Caputo fractional derivative is suitable enough for this purpose, as we shall see. Let us consider the linear FDE ${ }^{C} D_{0+}^{\alpha, \psi} N(t)=\lambda N(t)$, where $\alpha \in(0,1)$ is a real. The solution of the equation is given by the Mittag-Leffler function:

$$
N(t)=N_{0} E_{\alpha}\left(\lambda(\psi(t)-\psi(0))^{\alpha}\right) .
$$

Observe that, when $\alpha=1$, we recover the classical solution (15). We analyze growth rates in Lithuania and Qatar, countries with one of the lowest and the highest growth rates, respectively. The initial conditions are given by the initial population in each country: $N_{0}=2778550$, for Lithuania, and $N_{0}=47309$, for Qatar. In Figure 3, we present the obtained kernels for Lithuania and for Qatar that optimally fit with data. Figure 4 shows the two models (classical and fractional) for the two countries, compared with the population size. In Tables 1-2, we present relative errors obtained in the procedure and the values of the estimated parameters $\lambda$ and $\alpha$. 


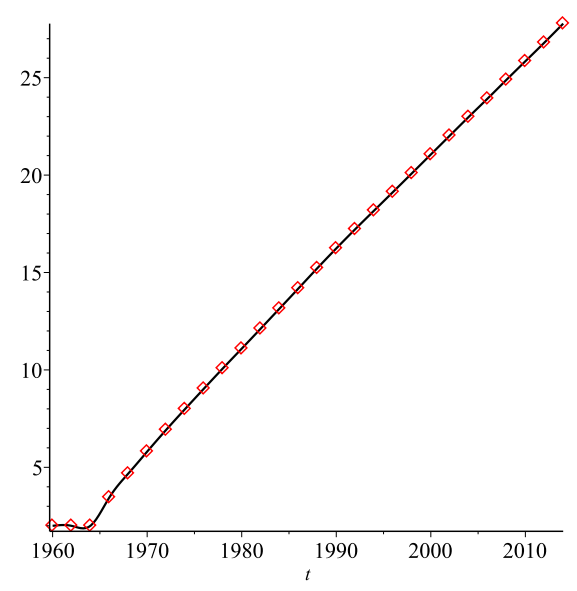

(a) Lithuania

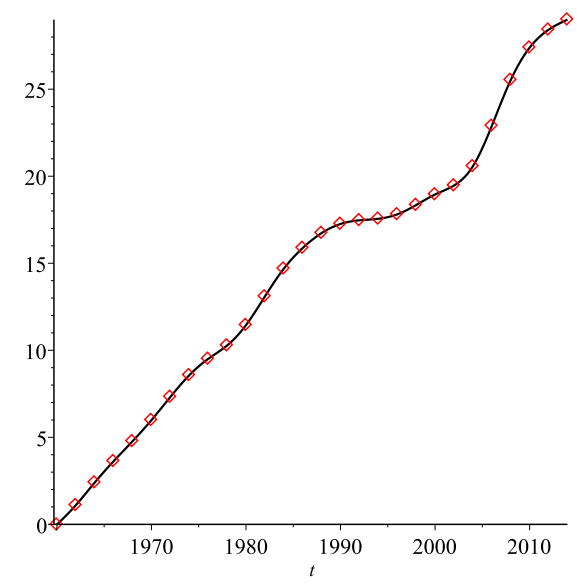

(b) Qatar

Figure 3. The optimal kernel for Lithuania and for Qatar.

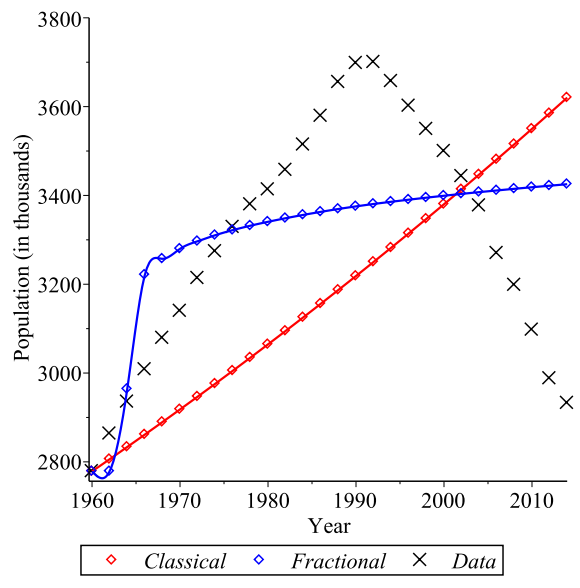

(a) Lithuania.

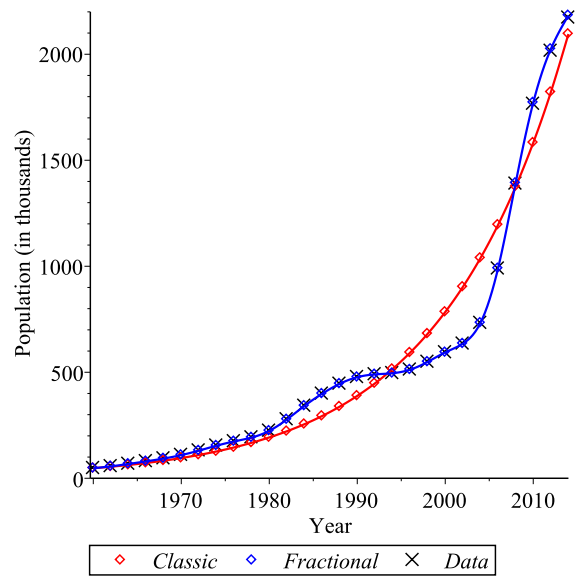

(b) Qatar

Figure 4. Population size: classical vs fractional models.

\begin{tabular}{ccccc}
\hline & Even years & Odd years & $\lambda$ & $\alpha$ \\
\hline Classical & 0.0104 & 0.6769 & 0.0049 & - \\
\hline Fractional & 0.0040 & 0.0045 & 0.1251 & 0.1111 \\
\hline
\end{tabular}

Table 1. Errors and the parameters with respect to Lithuania.

\begin{tabular}{ccccc}
\hline & Even years & Odd years & $\lambda$ & $\alpha$ \\
\hline Classical & 0.0217 & 0.0191 & 0.0702 & - \\
\hline Fractional & $6.5984 \times 10^{-10}$ & 0.0065 & 0.1503 & 0.9270 \\
\hline
\end{tabular}

Table 2. Errors and the parameters with respect to Qatar.

\subsection{GDP growth rate in USA}

The GDP per capita is equal to the GDP of a country divided by the midyear population of the country. This is the average per-person output of the economy. Kitov purposed in 2005 [16] a model to describe the GDP growth rate variations in the USA. He assumed that annual increment is constant in time and expressed the dynamics by a linear differential equation $G^{\prime}(t)=A$, where $G$ is the real GDP per capita and $A$ is a constant. The solution is given by

$$
G(t)=A t+G_{0}
$$


where $G_{0}$ is the starting GDP in the studied period. If we consider now the situation modeled by ${ }^{C} D_{0+}^{\alpha, \psi} G(t)=A$, its solution is given by the function

$$
G(t)=I_{0+}^{\alpha, \psi} A+G_{0}
$$

when $\alpha \in(0,1)$. Later, when we apply the least squares method to determine the optimal $\alpha$, we do not impose any constraint over $\alpha$ in order to obtain a better accuracy for the model. We also remark that, considering $\psi(t):=t$, and $\alpha \rightarrow 1^{ \pm}$, the fractional solution (18) converges to the classical solution (17). As initial condition we use $G_{0}=\$ 3007$, corresponding to the GDP per capita in USA in the year 1960. In Figure 5, we present the kernel obtained by the procedure, and compare both models to the given data.

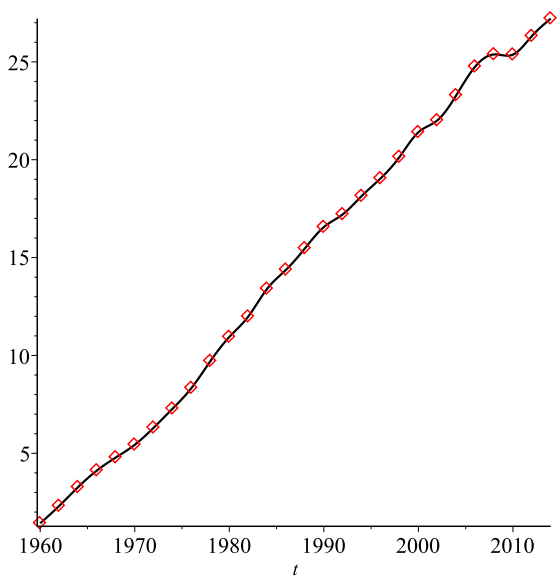

(a) Kernel.

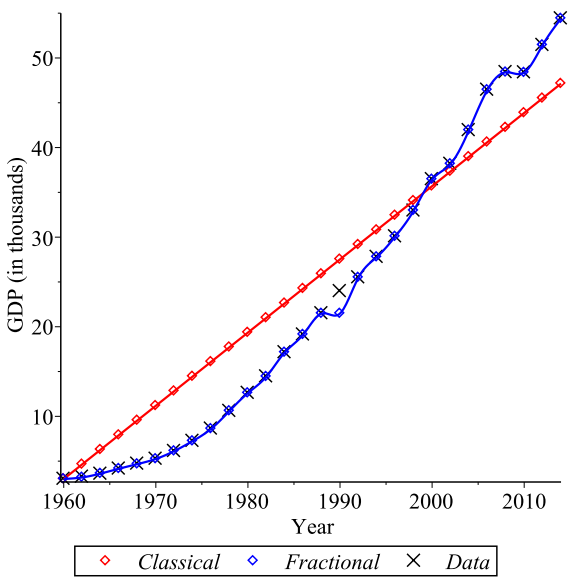

(b) GDP.

Figure 5. The optimal kernel and the GDP for USA.

In Table 3, we present the errors obtained, for the even and for the odd years, and the values of the parameters $A$ and $\alpha$.

\begin{tabular}{ccccc}
\hline & Even years & Odd years & $A$ & $\alpha$ \\
\hline Classical & 0.0299 & 0.0289 & 0.8172 & - \\
\hline Fractional & $3.0109 \times 10^{-7}$ & 0.0014 & 0.3287 & 1.6846 \\
\hline
\end{tabular}

Table 3. Errors and the parameters with respect to USA.

\section{Conclusions}

In recent years, many new types of fractional derivatives have been proposed, investigated and applied to real world models. Therefore, it is natural to try to combine those concepts into a single one. As we have mentioned, the important issue is to develop the fundamentals of a theory for fractional differential equations with a general derivative. In this paper, we have discussed the classical questions concerning differential equations, such as existence and uniqueness of solutions, the Picard iteration method and the long-term behaviour of solutions. Moreover, using the real data, we have shown, that mathematical models with the $\psi$-Caputo fractional derivative are more flexible. Apparently, the $\psi$-Caputo derivative has the potential to extract hidden aspects of real world phenomena.

\section{Acknowledgments}

R. Almeida was supported by Portuguese funds through the CIDMA - Center for Research and Development in Mathematics and Applications, and the Portuguese Foundation for Science and Technology (FCT-Fundação para a Ciência e a Tecnologia), within project UID/MAT/04106/2013; A. B. Malinowska by the Bialystok University of Technology grant S/WI/1/2016, and M. T. Monteiro by COMPETE: POCl-01-0145-FEDER-007043 and FCT-Fundação para a Ciência e a Tecnologia within the Project Scope: UID/CEC/00319/2013. The authors are very grateful to two anonymous referees, for valuable remarks and comments that improved this paper. 


\section{References}

1. E. Abad, S.B. Yuste and K. Lindenberg, Survival probability of an immobile target in a sea of evanescent diffusive or subdiffusive traps: A fractional equation approach. Phys. Rev. E 86 No 8 (2012) Article number 061120.

2. S. Abbas, G. Benchohra and M. N'Guérékata, Topics in Fractional Differential Equations, Developments in Mathematics 27, SpringerVerlag New York 2012.

3. Y. Adjabi, F. Jarad, D. Baleanu and T. Abdeljawad, On Cauchy problems with Caputo Hadamard fractional derivatives. J. Comput. Anal. Appl. 21 No 4 (2016) 661-681.

4. R. Almeida, A.B. Malinowska and T. Odzijewicz, Fractional differential equations with dependence on the Caputo-Katugampola derivative. J. Comput. Nonlinear Dynam. 11 No 6 (2016) Article number 061017.

5. R. Almeida, A Caputo fractional derivative of a function with respect to another function. Commun. Nonlinear Sci. Numer. Simul. 44 (2017) 460-481.

6. R. Almeida, N.R.O. Bastos and M.T.T. Monteiro, Modelling some real phenomena by fractional differential equations. Math. Meth. Appl. Sci. 39 No 16 (2016) 4846-4855.

7. A. Alsaedi, J.J. Nieto and V. Venktesh, Fractional electrical circuits. Adv. Mech. Eng. 17 No 11 (2015) Article number 1687814015618127.

8. R. Bellman, Stability Theory of Differential Equations. McGraw Hill, New York, 1953.

9. T.A. Burton, Fractional equations and a theorem of Brouwer-Schauder type. Fixed Point Theory 14 No 1 (2013) 91-96.

10. K. Diethelm, The Analysis of Fractional Differential Equations. Lecture Notes in Mathematics, Springer-Verlag, Berlin, 2010.

11. Y.Y Gambo, F. Jarad, D. Baleanu and T. Abdeljawad, On Caputo modification of the Hadamard fractional derivatives. Adv. Difference Equ. 2014:10 (2014) doi:10.1186/1687-1847-2014-10.

12. Z. Gong, D. Qian and C. Li P. Guo, On the Hadamard Type Fractional Differential System, in Fractional Dynamics and Control (eds D. Baleanu, J. A. T. Machado and A. C. J. Luo), 159-171 Springer New York, 2012.

13. R. Hilfer, Applications of Fractional Calculus in Physics. World Scientific Publishing Co, Singapore, 2003.

14. F. Jarad, T. Abdeljawad and D. Baleanu, Caputo-type modification of the Hadamard fractional derivatives. Adv. Difference Equ. 2012:142 (2012) doi:10.1186/1687-1847-2012-142.

15. A.A. Kilbas, H.M. Srivastava and J.J. Trujillo, Theory and Applications of Fractional Differential Equations. North-Holland Mathematics Studies, 204. Elsevier Science B.V., Amsterdam, 2006.

16. I. Kitov, GDP growth rate and population. Economics Bulletin 28 (9) (2005) pages A0.

17. S. Liu, H. Li, Q. Dai and J. Liu, Existence and uniqueness results for nonlocal integral boundary value problems for fractional differential equations. Adv. Difference Equ. 2016:1 (2016) doi=10.1186/s13662-016-0847-x.

18. Y. Luchko and J.J. Trujillo, Caputo-type modification of the Erdélyi-Kober fractional derivative. Fract. Calc. Appl. Anal. 10 (3) (2007) 249-267.

19. R. L. Magin, Fractional Calculus in Bioengineering. Begell House Inc. Publisher, 2006.

20. C. Moler, J. Little and S. Bangert, Matlab User's Guide - The Language of Technical Computing, The MathWorks, Sherborn, Mass. (2001).

21. S. G. Samko, A. A. Kilbas and O. I. Marichev, Fractional integrals and derivatives, translated from the 1987 Russian original. Gordon and Breach, Yverdon, 1993.

22. R.T. Sibatov and V.V. Uchaikin, Fractional differential approach to dispersive transport in semiconductors. Phys. Uspekhi 52 No 10 (2009) 1019-1043.

23. The World Bank. World DataBank http://databank.worldbank.org/. Accessed 16 October 2016.

24. F.G. Tricomi and A. Erdélyi, The asymptotic expansion of a ratio of gamma functions. Pacific J. Math. 1 No 1 (1951) $133-142$.

25. R. Yan, S. Sun, Y. Sun and Z. Han, Boundary value problems for fractional differential equations with nonlocal boundary conditions Adv. Difference Equ. 2013 No 1 (2013) 176.

26. D. Yau, A First Course in Analysis. World Scientific Publishing Company, 2013.

27. H. Ye, J. Gao and Y. Ding, A generalized Gronwall inequality and its application to a fractional differential equation. J. Math. Anal. Appl. 328 (2007) 1075-1081.

28. Y. Yu, P. Perdikaris and G.E. Karniadakis, Fractional modeling of viscoelasticity in 3D cerebral arteries and aneurysms. J. Comput. Phys. 323 (2016) 219-242. 